\title{
Corneal Biomechanical Properties Characterization Using Air-jet Indentation Based Optical Coherence Tomography System (AIOCT)
}

\author{
Li-ke Wang ${ }^{1}$, Jia-ying Zhang ${ }^{1,2,3}$, Tian-jie $\mathrm{Li}^{1}$, Xue-yong Zhang ${ }^{4}$, Lei Tian ${ }^{1,5}$ and Yong-ping Zheng ${ }^{1}$ \\ ${ }^{1}$ Department of Biomedical Engineering, The Hong Kong Polytechnic University, Hong Kong 999077, China \\ ${ }^{2}$ Department of Laser Medicine, Chinese PLA General Hospital, Beijing 100853, China \\ ${ }^{3}$ Eye Hospital, China Academy of Chinese Medical Sciences, Beijing 100040, China \\ ${ }^{4}$ School of Mathematics and Physics, Anhui Jianzhu University, Hefei 230601, China \\ ${ }^{5}$ Beijing Tongren Eye Center, Beijing Ophthalmology \& Visual Sciences Key Laboratory, Beijing Tongren Hospital, Capital Medical \\ University, ophthalmology Beijing, Beijing, 100730, China
}

\begin{abstract}
We proposed a novel method together with system to quantify the corneal biomechanics. The objectives of this study are to develop a method to measure the corneal biomechanical properties in vivo and to evaluate the performance of AIOCT system. A novel AIOCT system was established and a mathematical model was built on the basis of the current air-jet indentation method. Experiments were performed to evaluate the mechanical properties of eleven custom-made silicone corneal mimicking phantoms by the AIOCT system under different intraocular pressures (IOP). The results were then compared with those measured by the standard mechanical tensile test and indentation test. The phantom's moduli were ranged from 0.08 to $1.03 \mathrm{MPa}$ according to the tensile and indentation test. The elastic moduli of corneal silicone phantoms $\left(E_{\text {airjet }}\right)$ were measured using the AIOCT system with the proposed mathematical model at four IOPs $(0.6,15.3,29.8$, and $44.7 \mathrm{mmHg})$. The $E_{\text {airjet }}$ were agreed well with the corresponding moduli $\left(E_{\text {tensile }}\right)$ measured by the tensile test $\left(E_{\text {airjet }}=1.188 E_{\text {tensile }}, R^{2}=0.925\right)$. The preliminary results showed that this technique was capable of measuring corneal biomechanical properties in vivo and it may provide a clinical potential non-invasive and non-contact means to the diagnosis of keratoconus suspects in the future.
\end{abstract}

\section{Introduction}

Corneal biomechanical properties are shown to be correlated with the pathologies of corneal degeneration diseases like keratoconus, keratectasia, pellucid marginal degeneration [1,2] and glaucoma [3, 4]. However, there is still impossible to measure the corneal properties in vivo due to the absent of clinical available method. The absent of available method to measure the corneal biomechanical properties in vivo leads to a serious adverse effect, for example, to the clinical diagnosis accuracy, and to optimize the treatment or to predict the treatment effectiveness and efficiency.

Keratoconus is a common corneal dystrophy characterized by the progressive thinning of central and para-central stroma, and the subsequent conical ectasia. It leads to irregular astigmatism, corneal scaring, keratocytes apoptosis and even blindness. Recently, the iatrogenic keratoconus after Laser-Assisted in Situ Keratomileusis (LASIK) surgery has been paid much attention [5]. Due to the cutting of stroma, keratoconusis may irritate immediately, months or years after the surgery and the irritation most likely occur among the preoperative keratoconus suspects. The effective and timely differentiation of suspected corneas before operation and scientific strategies management for such patients is of great interest to decrease the complication of LASIK and to avoid the irreversible vision loss of these people. Current clinical diagnosis of keratoconus depends mainly on corneal pachymetry and tomography by which typical cases of keratoconus instead of the suspects are screened out $[6,7]$. In keratoconus patient's stroma, the collagen fibrils and interfibrillary substance loss and slippage lead to biomechanical instability of cornea and subsequently cause alteration of the corneal tomography $[8,9]$. The changes in corneal biomechanical properties are usually occurred before the topographic abnormalities, like the majority of tissue pathologies. Thus, the quantitative clinical measurement of corneal biomechanical properties in vivo will make a significant difference in the early and sensitive diagnosis preoperatively on keratectasia suspects whose corneas are softer than normal, but corneal thickness and tomography would otherwise be within the normal criteria for surgery. Furthermore, considering such a complication can occur months or even years after the surgery $[10,11]$, the prompt diagnosis during the official visits is helpful for early interventions on the cases.

Corneal biomechanics is a relatively new concept compare to central corneal thickness (CCT). The collagen 
chemical composition and structure of corneal stroma are the basis governing the corneal biomechanical properties $[12,13]$ due to the high tensile stiffness of collagen fibrils and spatially varying distribution of lamellae in the stroma. Cornea is a tissue with nonlinear, anisotropic, heterogeneous, and viscoelastic characteristics, both laterally and by depth [14]. Evaluations on corneal biomechanical properties include the measurements of corneal rigidity, viscosity and elasticity. Abundant efforts have been devoted into the development of measuring techniques on corneal biomechanical properties, for example, in vitro traditional uniaxial tensile tests [15], inflation experiments [16, 17], and nano indentation based atomic force microscopy [18]. Most of them measure the average of the gross corneal biomechanical properties and all fail to be used in clinic due to the destruction of cornea. The investigations on isolated corneal strips are limited, due to the inappropriate evaluation of corneal natural curved condition. The experimental results on the isolated cornea are also hard to control due to the significant effects of corneal hydration [2, 19]. Several potential in vivo measurements are subsequently developed, including the opto-mechanical testing device [20], mechanical corneal indentation device $[21,22]$, high-frequency ultrasound elasticity microscope [23, 24], novel scanning acoustic microscopy [25], and confocal Brillouin microscopy [26]. Among them, topical ocular anesthesia is required for the mechanical testing device and eye immersion is required for ultrasound technique which makes inconvenience of clinical application. The Brillouin microscopy cannot access corneal dynamic properties in its current form.

Ocular Response Analyzer (ORA; Reichert, Corp., Buffalo, NY) is the first instrument that allows the evaluation of corneal biomechanical properties in vivo. It is one of the popular clinical minorities to measure corneal biomechanical properties, including, corneal hysteresis $(\mathrm{CH})$ and corneal resistance factor (CRF), with $\mathrm{CH}$ quantifying the corneal viscoelastic mechanical damping ability whereas CRF indicating the whole corneal viscoelastic resistance ${ }^{27}$. While given the viscoelasticity and poroelasticity of cornea, the interpretation of ORA data is difficult. And its sensitivity and efficiency in identifying keratoconus suspects or evaluation of cross-linking (CXL) effect, the recent most popular intervention to reverse keratoconus, were questioned a lot [27-29]. Newer Corneal Visualization with Scheimpflug Technology (Corvis ST; Oculus Inc., Wetzlar, Germany) based on Scheimpflug imaging technique, has become another method for measuring corneal biomechanical properties in clinic since 2010 . The corneal displacement amplitude during an air-puff indentation holds promise to yield relevant parameters related to the corneal biomechanical properties, however, at present, none of the parameters from Corvis ST can be deemed directly as corneal biomechanical properties [30]. Theoretically, Scheimpflug imaging technique is limited by geometrical and optical distortions which both make the careful correction necessary beyond the quantitative extraction of biomechanics related information [31-33].

In this decade, optical coherence tomography (OCT) system has been widely applied to ophthalmologic imaging for visualizing both anterior and posterior segments of the eye in clinic [34-36]. Most recently, OCT is used in corneal biomechanical properties detection as novel and developed optical means for its progressive resolution (about a dozen of micrometers along the longitudinal axis and twenty micrometers in the transversal axis). In 2011, OCT was at the first time to integrate with air puff for the measurement of the corneal biomechanical properties [37]. The trends were illustrated with OCT that stiffened cornea after CXL led to a smaller corneal deformation under the same air-puff and the cornea presented stiffer (higher elastic modulus) when intraocular pressure (IOP) was higher [38]. Further studies are needed to verify the potential clinical meaning of this new instrument and method, and deduce the corneal elastic modulus. Meanwhile, considering orders of magnitude of differences among the reported corneal elastic modulus (0.159 $\mathrm{MPa}$ [17] to $57 \mathrm{MPa}$ [2]) with varieties of techniques, methods and corresponding mathematical models should be further improved based on the reasonable and reliable input data from OCT measurement to extract the quantitative corneal elastic modulus and the relationships among structure, biomechanics, and optical performance of cornea in physiological or pathological conditions, especially preand post-keratore fractive surgery.

In the present study, an Air-jet Indentation based Optical Coherence Tomography (AIOCT) system was established to integrate the air-jet system into a custom-made spectral-domain OCT system. Air-jet is used as an external excitation to induce corneal deformation, while the spectral-domain OCT system is used to monitor and record the real-time corneal dynamic deformation. Eleven cornea-mimicking silicon phantoms were designed to validate the performance of AIOCT system on the measurement of corneal biomechanical properties and avoid the limitations during the measurement on cornea in vitro as being mentioned above. The corneal mimicking phantoms were fabricated according to the reported data on corneal shape, geometries and elasticity, especially those considering the nuance of elasticity under physiological and pathological situations. Accordingly, the cross-sectional images of the corneal phantoms under different set IOPs were recorded during the whole process of air-jet corneal indentation/relaxation. Meanwhile, a mathematical model was proposed on the basis of the current air-jet indentation method to determine the corneal elastic modulus based on the biomechanical parameters of silicon corneal phantoms detected by OCT. The measurement repeatability of the AIOCT system was also evaluated.

\section{Materials and methods}

\subsection{AIOCT system establishment and calibration}

The Air-jet Indentation based spectral-domain OCT (AIOCT) system contains a super luminescent diode (SLD) with a central wavelength of $850 \mathrm{~nm}$ and light power of $4.0 \mathrm{~mW}$. The system schematic of the developed 
system for measuring the corneal biomechanical properties in vivo is shown in Fig.1. An air-jet component is integrated to the system and the air-jet is used as an external excitation to deform the cornea for $15 \mathrm{~ms}$. Meanwhile, spectral domain OCT system is used to monitor the dynamic response of the cornea under air-jet indentation using transient M-mode at a speed of $24 \mathrm{~K}$ A-scans per second. The depth of observation is $2.7 \mathrm{~mm}$ along the longitudinal direction of the cornea and can cover the entire cornea during the dynamic corneal deformation. Both the waveforms of air-jet and corneal dynamic deformation are recorded for post measurement analysis to further assess the corneal mechanical properties. A CCD camera is integrated with the system for facilitating the alignment control of testing site. A calibrated pressure sensor (PMP 1400, GE Druck, Leicester, England) is used for measurement of the generated air-jet pressure. The valve of the pressures senor is connected to air-jet nozzle with a tube. Before the elasticity measurement, apex of a corneal phantom is aligned with the air-jet nozzle and is positioned at $11 \mathrm{~mm}$ away from the air-jet nozzle (Fig.1).

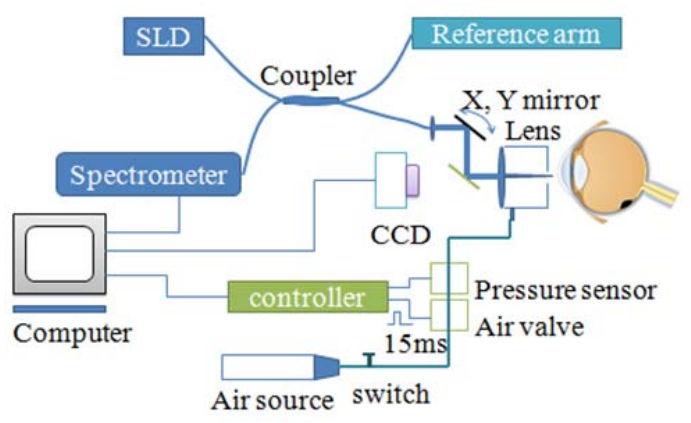

Fig. 1. Schematic of the AIOCT system for corneal biomechanical properties in vivo measurement.

\subsection{Silicone corneal model and measurement of corneal elasticity}

To represent the natural condition of cornea and avoid the effect of corneal hydration ex vivo on the intervention of experimental results, silicone-moulded corneal mimicking phantoms were designed and fabricated in the present study. In order to mimic the natural condition of cornea, the corneal phantoms were fabricated with the similar shape of human cornea and the different elasticity according to previous study [39] (Fig.2 a). The corresponding silicone matrixes (Fig.2 b) under different elasticity were applied in the tensile test and mechanical indentation test for instrument calibration.

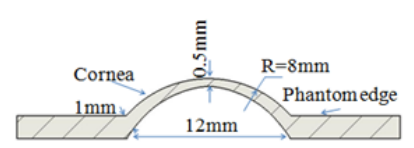

(a)

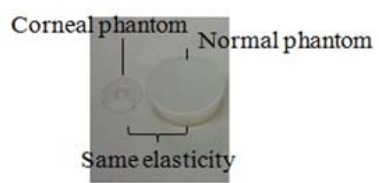

(b)
Fig. 2. (a) Schematic of silicone corneal phantom, and (b) fabricated silicone corneal phantom and silicone matrix (normal phantom) with the same elasticity.

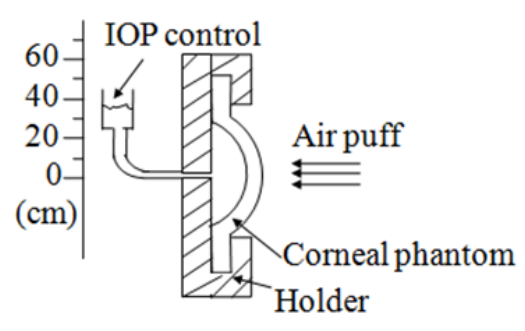

Fig. 3. Schematic of the silicone corneal phantom connected with liquid reservoir for IOP control.

The silicone corneal phantom was mounted in a customized metal holder with the artificial anterior chamber filled with liquid from the connected syringe (Fig.3). Different IOPs were realized by adjusting the height of the connected syringe and the IOP was measured by a calibrated pressure sensor (OPP-M400, Opsens Inc., Canada) inserted into the connected vessel system at the same level. Four IOPs $(0.6,15.3,29.8$, and $44.7 \mathrm{mmHg}$ ) were applied to the corneal phantom successively and measurements were taken under the real-time A-line scan image guidance. Information of the initial thickness, the maximum of air pressure in the AIOCT system and the maximum deformation of the anterior surface under different IOP conditions were extracted from the OCT images (Fig.4).

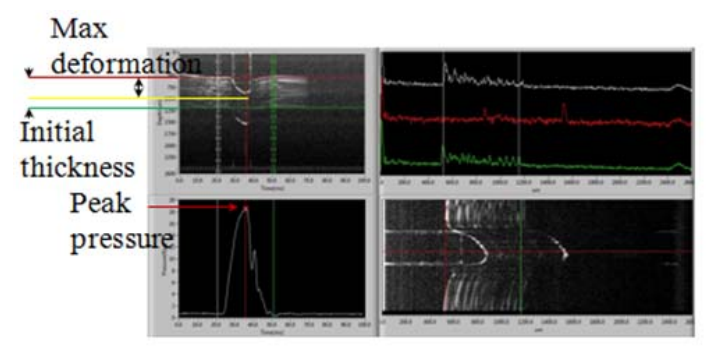

Fig. 4. Corneal deformation profile and air-jet pressure profile recorded by the AIOCT system. Initial corneal thickness, maximum air-jet pressure in AIOCT and maximum corneal deformation amplitude can be extracted from the recorded profiles.

\subsection{Air-jet indentation using AIOCT}

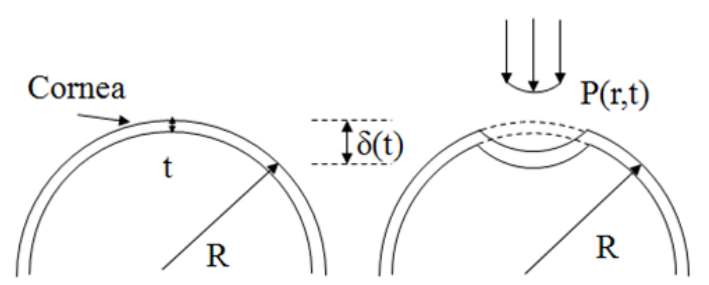

Fig. 5. Schematic of the corneal deformation under an air-jet indentation.

The measurement of corneal elastic modulus is based on corneal geometries and the recorded corneal dynamic deformation under the air-jet indentation. An external air-jet pressure $P(t)$ is applied on the corneal surface 
(Fig.5) and the distribution of the air-jet pressure on the corneal surface can be expressed by the following equation [40],

$$
P(\mathrm{r})=P_{\max } \exp ^{-0.44 r^{2}}
$$

Where $r$ is the radius of air-jet pressure from its centerline, the mean pressure applied on cornea can be calculated as follow,

$$
\bar{P}=\frac{\int_{0}^{2 \pi} \int_{0}^{R} P_{\max } \cdot e^{-0.44 r^{2}} r d r d \theta}{\pi R^{2}}=\frac{2 P_{\max }}{R^{2}}\left(\frac{1-\mathrm{e}^{-0.44 R^{2}}}{0.88}\right)
$$

where $\mathrm{R}$ is the radius of deformation area. Considering the small deformation of cornea, $\mathrm{R}$ can be replaced as $R^{2} \approx 2 R_{c} \cdot D A . R_{c}$ is the curvature of corneal radius and $D A$ is the deformation amplitude of cornea. The corneal elastic modulus can be simplified by dividing the change of strain by the change of stress after calculation of stress [41] and strain [42],

$$
\begin{aligned}
E & =\frac{\Delta s \operatorname{tress}}{\Delta s \operatorname{train}} \\
& =(1-v) \frac{R_{c} \bar{P} / 2 t}{D A / R_{c}} \\
& =(1-v) \frac{R_{c}{ }^{2}}{2 t} \cdot \frac{\bar{P}}{D A}
\end{aligned}
$$

The $P_{\max }$ and DA can be obtained by AIOCT system, and the central corneal thickness ( $\mathrm{t}$ ) and corneal curvature of cornea can be measured before test. The air-jet indentation elastic modulus $\left(E_{\text {airjet }}\right)$ can then be calculated by solving the equation (3).

\subsection{Calibration and measurement of the air-jet pressure on the corneal surface}

The air-jet pressure $P(t)$ in the mathematical model is the air-jet pressure on the corneal surface. A detailed calibration of air pressure in the AIOCT system was performed with a pressure sensor (MPXV5100GC6U, Freescale Semiconductor Inc., Tempe, AZ, USA). The air-jet pressure on the corneal surface was kept $11 \mathrm{~mm}$ away from the air-jet nozzle of AIOCT system. A calibration chart was obtained for the conversion of the air pressure to the maximum air-jet pressure $\left(P_{\max }\right)$ for equation (3).

\subsection{Mechanical testing}

Eleven silicone matrixes were tested with a flat-end cylindrical indenter (4 $\mathrm{mm}$ diameter) using mechanical testing machine (Instron 5569, Norwood, MA, USA) (Fig.6 a). The elastic modulus of phantom is extracted from force-deformation relationship based on the indentation model [43],

$$
E=\frac{\left(1-v^{2}\right)}{2 a \kappa(v, a / h, \omega / h)} \cdot \frac{F}{\omega},
$$

where $F$ is the indentation force, $a$ is the radius of the indenter, $\omega$ is the indentation depth, $h$ is the initial thickness of phantom, and $v$ is the Poisson's ratio of phantom. $\kappa$ is a geometric and material-dependent correction factor, and is a function of the value of $v, a / h$ and $\omega / h$. The Poisson's ratio of the phantoms is assumed to be a constant value of 0.45 , which indicated a near incompressibility of the tested phantom. $F / \omega$ can be obtained using linear regression between the indentation force and deformation with a maximum deformation of $15 \%$ of the initial phantom thickness.

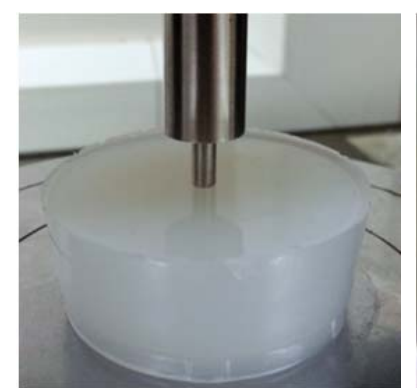

(a)

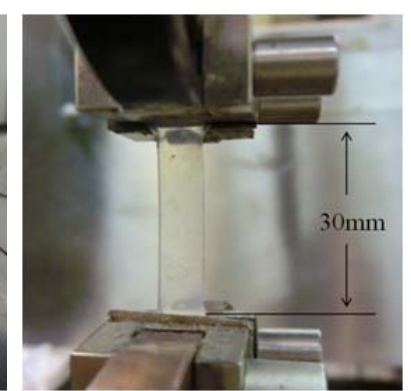

(b)
Fig. 6. Mechanical testing setups for silicone matrix and strip, respectively. (a) mechanical indentation test and (b) uniaxial tensile test.

After the mechanical indentation test, the eleven silicone matrixes were cut into $5 \mathrm{~mm}$ wide by $35 \mathrm{~mm}$ long uniform strips. Strips were then tested with tensile test using a universal testing machine (Alliance RT/5, MTS Corporation, MN, USA) (Fig. 6 b). The strips were pre-stressed by placing samples with initial length of 30 $\mathrm{mm}$ under slight tension and then tested to $30 \%$ strain. Elastic modulus of the strip can then be determined using the equation [44],

$E=\frac{\text { true stress }}{\text { trues train }}=\frac{T(1+\zeta / L) / A}{\ln (1+\zeta / L)}$,

where $T$ is the axial load and $\zeta$ is the specimen elongation, $L$ is the initial length of the strip specimen and $A$ is the initial cross-sectional area.

Elastic modulus of the matrixes and corneal phantoms measured by both mechanical tensile and indentation were compared. The measured $E_{\text {airjet }}$ results from AIOCT test at different IOPs were compared with the elastic modulus obtained by tensile test $\left(E_{\text {tensile }}\right)$ at the corresponding stress levels that equivalent to the set IOPs.

\section{Statistical analysis}

Statistical analyses were performed using SPSS version 20 (SPSS Inc., Chicago, IL, USA). Three measurements were obtained using AIOCT system by a single operator. The within-subject standard deviation ( $\mathrm{Sw})$, repeatability $(2.77 \mathrm{x} \mathrm{Sw})$, coefficient of variation $(\mathrm{CV})$ and intra-class correlation coefficient (ICC) were analyzed. Good measurement repeatability required an ICC of above 0.75 [45]. A $p$ value $<0.05$ was considered as statistically significant in this study.

\section{Results}




\subsection{Fabrication of the silicone corneal phantoms}

Eleven silicone corneal mimicking phantoms with different elasticity were fabricated with the initial central corneal thickness of $504.12 \pm 17.04 \mu \mathrm{m}$. There was no significant difference among neither different peripheral points of the same model, nor the center points of different models $(p>0.05)$.

\subsection{Phantoms measurement using mechanical test}

The elastic moduli of the corneal phantoms measured by tensile test ranged from 0.08 to $1.03 \mathrm{MPa}$, which is in the similar range of human cornea as reported in literature $[15,46]$. The tensile elastic modulus ascertained at $3 \%$ strain $\left(E_{\text {tensile }}\right)$ was compared with indentation elastic modulus $\left(E_{\text {indentation }}\right)$ in Fig.7. The comparable results showed a good consistency of the two standard methods $\left(E_{\text {tensile }}=1.022 E_{\text {indentation }}, R^{2}=0.995\right)$.

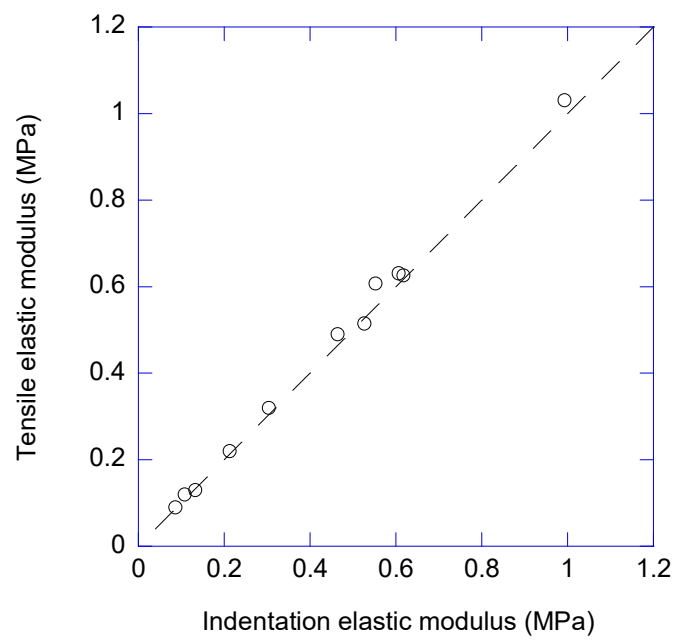

Fig. 7. Comparison of elastic moduli measured using tensile test and indentation test $\left(E_{\text {tensile }}=1.022 E_{\text {indentation }}, R^{2}=0.995\right)$.

\subsection{Phantoms measurement using AIOCT system}

The Sw, repeatability and CV of the AICOT measurement was shown in Table 1. The intraclass correlation coefficient (ICC), which assessed measurement repeatability, of three consecutive measurements of the air-jet elastic modulus $\left(E_{\text {airjet }}\right)$ on corneal phantom showed a high value of 0.951 (95\%CI: $0.921-0.972)$. Fig.8 compared the $E_{\text {airjet }}$ measured by AIOCT system under different IOPs (i.e. 0.6, 15.3, 29.8 and $44.7 \mathrm{mmHg}$ ) and the $E_{\text {tensile }}$ measured by tensile test at the corresponding stress levels. The result showed that the $E_{\text {airjet }}$ agreed well with $E_{\text {tensile }}$ at different IOPs level $\left(E_{\text {airjet }}=1.188 E_{\text {tensile }}\right.$, $\left.R^{2}=0.925\right)$.

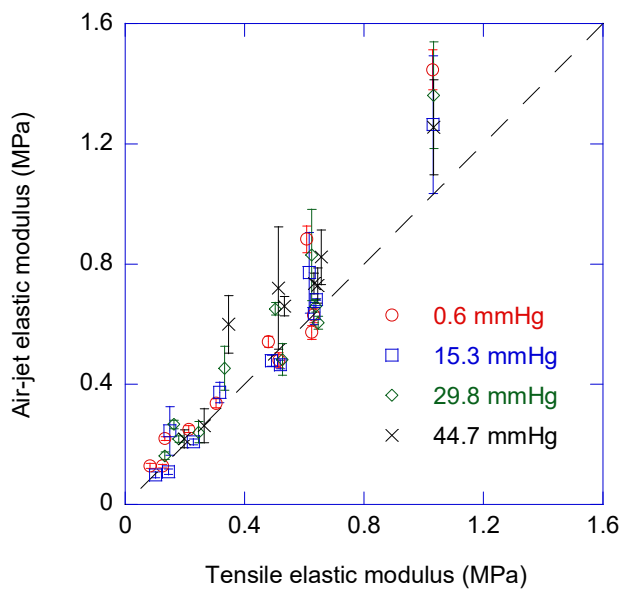

Fig. 8. Comparison of the elastic moduli measured by AIOCT system under set IOPs of $0.6 \mathrm{mmHg}$ (red circle; $E_{\text {airjet }}=1.210$ $E_{\text {tensile, }}, R^{2}=0.906$ ), $15.3 \mathrm{mmHg}$ (blue square; $E_{\text {airjet }}=1.119$ $E_{\text {tensile }}, R^{2}=0.954$ ), $29.8 \mathrm{mmHg}$ (green diamond; $E_{\text {airjet }}=1.188$ $E_{\text {tensile }}, R^{2}=0.920$ ) and $44.7 \mathrm{mmHg}$ (black cross; $E_{\text {airjet }}=1.233$ $\left.E_{\text {tensile }}, R^{2}=0.935\right)$, and the elastic moduli of the corresponding phantom matrix measured by tensile test $(\mathrm{n}=11$; for all pressures, $E_{\text {airjet }}=1.188 E_{\text {tensile }}, R^{2}=0.925$ ).

Table 1. The within-subject standard deviation $(\mathrm{Sw})$, repeatability $(2.77 \mathrm{x}$ Sw), coefficient of variation $(\mathrm{CV})$ for the AIOCT measurement.

\begin{tabular}{lllll}
\hline$E_{\text {airjet }}$ & $\begin{array}{l}\text { Sw } \\
(95 \% \mathrm{CI})\end{array}$ & $\begin{array}{l}\text { Repeatability } \\
(95 \% \mathrm{CI})\end{array}$ & $\begin{array}{l}\mathrm{CV}(\%) \\
(95 \% \mathrm{CI})\end{array}$ & $\begin{array}{l}\text { ICC } \\
(95 \% \mathrm{CI})\end{array}$ \\
\hline $0-0.4 \mathrm{MPa}$ & $\begin{array}{l}0.022 \mathrm{MPa} \\
(0.012-0.032)\end{array}$ & $\begin{array}{l}0.061 \mathrm{MPa} \\
(0.032-0.090)\end{array}$ & $\begin{array}{l}9.8 \\
(5.9-13.7)\end{array}$ & $\begin{array}{l}0.856 \\
(0.705-0.942)\end{array}$ \\
& & & & \\
\hline \multirow{2}{*}{$0.4-0.8 \mathrm{MPa}$} & $0.048 \mathrm{MPa}$ & $0.133 \mathrm{MPa}$ & $(4.7-10.8)$ & $(0.389-0.824)$ \\
& $(0.026-0.070)$ & $(0.071-0.194)$ & & \\
\hline \multirow{2}{*}{$0.8-1.2 \mathrm{MPa}$} & $0.131 \mathrm{MPa}$ & $0.363 \mathrm{MPa}$ & 11.8 & 0.759 \\
& $(0.082-0.180)$ & $(0.228-0.498)$ & $(7.7-15.9)$ & $(0.375-0.948)$ \\
\hline \multirow{2}{*}{ All } & & & & \\
& $0.052 \mathrm{MPa}$ & $0.144 \mathrm{MPa}$ & 9.2 & 0.951 \\
& $(0.034-0.069)$ & $(0.095-0.192)$ & $(7.0-11.3)$ & $(0.921-0.972)$
\end{tabular}

\section{Discussion and conclusion}

Studies reported that only one hour large elevation of IOP made sub-micron scale changes in cornea, i.e. the 
collagen fibrils thinned and disorientated, which may be a substantial basis for the higher stiffness manifested in cornea suffered from enhanced IOP [47]. Resulted from the dangers in altering IOP of human eyes in vivo, most of the previous studies were confined to measure the overall biomechanical properties and the physiological changes of cornea under different experimental conditions in vitro. So the conclusive controversy existed. To minimize such limitations, silicone corneal mimicking phantoms were designed and fabricated to mimic the human corneal shape, geometries and elasticity. These phantoms were further applied to test the repeatability of the developed AIOCT system with the proposed mathematical modeling for corneal elastic modulus measurement.

Corvis ST and ORA are the only commercial available clinical device that can assess the corneal biomechanical properties. Given the mechanism of the ultra-high-speed Scheimpflug imaging in Corvis ST and our observation of the obvious flutter in its corneal images, the reliability of Corvis on the data of DA was doubted, and the test-retest variability of DA by Corvis was shown to be low [48]. On the contrary, the optical measuring mechanism of OCT provided a more accurate way to monitor the corneal dynamic deformation. In ORA, large corneal dynamic deformations are induced over a period of about $25 \mathrm{~ms}$ and two parameters of corneal hysteresis $(\mathrm{CH})$ and corneal resistance factor $(\mathrm{CRF})$ were issued. However, the interpretations of the parameters are difficult which makes ORA not effective in practice when assessing the corneal biomechanical properties, and differentiating the keratoconus suspects from either LASIK candidates or subjects on official visit postoperatively.

In this study, an Air-jet Indentation based Optical Coherence Tomography (AIOCT) system was established to integrate the air-jet system with custom-made spectral-domain OCT system for the measurement of corneal biomechanical properties in vivo. The air-jet were used as an external excitation to induce corneal deformation, while a custom made spectral-domain OCT system is used to monitor and record the corneal dynamic deformation. A novel mathematical model for the calculation of corneal elastic modulus on the basis of the current air-jet indentation method was deduced. The AIOCT system demonstrated a good measurement repeatability $(\mathrm{ICC}=0.951)$ and good corneal elastic modulus measurement agreement with the tensile test $\left(\mathrm{R}^{2}\right.$ $=0.925)$. The AIOCT provided a promised clinical strategy to quantitatively measure the corneal biomechanical properties. The current AIOCT measurement is non-contact, high speed and high resolution, which may provide a great contribution to clinic. Combination of the air-jet indentation and the OCT based system provides a novel elasticity measurement with high precision. The diameter of the AIOCT's air-jet nozzle is $2 \mathrm{~mm}$ which is smaller than that the nozzle diameter $(2.5 \mathrm{~mm})$ of Corvis ST and ORA. Although the air-jet component of the three instruments all imply that, not all the corneal mass involves in the dynamical motion, a more localized corneal biomechanical properties are measured by the current AIOCT system. Clinical measurement of corneal biomechanical properties in vivo provides a useful tool for better understanding of keratoconus, quantitative assessment of CXL treatment efficiency and role of cornea in glaucoma development and progression. It can also prevent LASIK ectasia and ultimately improves LASIK outcomes through exact screening among LASIK candidates. In the future, the elasticity map can be drawn if the measurement point is successful to be moved around the cornea, accompanied with more localized measurement and refined measuring method.

In conclusion, this study developed the AIOCT system and mathematical model for the measurement of corneal elastic modulus in vivo. Corneal phantom experiments demonstrated the AIOCT measurement has a good repeatability and good measurement agreement with the standard mechanical test. It is prospectively helpful in clinical screening of keratoconus suspects in the future.

\section{Acknowledgement}

This work was partially supported by grants of the National Natural Science Foundation of China (61471003), the Hong Kong Polytechnic University Joint $\mathrm{PhD}$ Supervision Scheme (G-UB58), Beijing Natural Science Foundation (7174287). The authors are grateful for the inputs of Dr. Match Ko for his contributions to the study and the supports from Dr. Andrew KC Lam and Miss Shanica Y Hon, the researcher of School of Optometry, The Hong Kong Polytechnic University. No conflict of interest exists.

\section{References}

1 D. Ortiz, D. Piñero, M. H. Shabayek, F. Arnalich-Montiel, and J. L. Alió, Journal of Cataract \& Refractive Surgery 33, 1371 (2007).

2 T. T. Andreassen, A. Hjorth Simonsen, and H. Oxlund, Experimental eye research 31, 435 (1980).

3 C. Johnson, S. Mian, S. Moroi, D. Epstein, J. Izatt, and N. Afshari, Investigative ophthalmology \& visual science 48, 2540 (2007).

4 M. W. L. Ko, JSM Ophthalmology (2014 (under review)).

5 T. Seiler and A. W. Quurke, Journal of Cataract \& Refractive Surgery 24, 1007 (1998).

6 R. Ambrósio Jr, R. S. Alonso, A. Luz, and L. G. Coca Velarde, Journal of Cataract \& Refractive Surgery 32, 1851 (2006).

7 A. Konstantopoulos, P. Hossain, and D. F. Anderson, British Journal of Ophthalmology 91, 551 (2007).

8 K. M. Meek, S. J. Tuft, Y. Huang, P. S. Gill, S. Hayes, R. H. Newton, and A. J. Bron, Investigative ophthalmology \& visual science 46, 1948 (2005).

9 A. Gefen, R. Shalom, D. Elad, and Y. Mandel, Journal of the mechanical behavior of biomedical materials 2, 224 (2009).

10 J. D. Marsack, K. E. Parker, Y. Niu, K. Pesudovs, B. Optom, and R. A. Applegate, Journal of Refractive Surgery 23, 960 (2007). 
11 M. A. Woodward, J. B. Randleman, B. Russell, M. J. Lynn, M. A. Ward, and R. D. Stulting, Journal of Cataract \& Refractive Surgery 34, 383 (2008).

12 D. W. DelMonte and T. Kim, Journal of Cataract \& Refractive Surgery 37, 588 (2011).

13 M. Winkler, D. Chai, S. Kriling, C. J. Nien, D. J. Brown, B. Jester, T. Juhasz, and J. V. Jester, Investigative ophthalmology \& visual science 52, 8818 (2011).

14 W. Kokott, Graefe's Archive for Clinical and Experimental Ophthalmology 138, 424 (1938).

15 G. Wollensak, E. Spoerl, and T. Seiler, Journal of Cataract \& Refractive Surgery 29, 1780 (2003).

16 J. Ø. Hjortdal, Journal of biomechanics 29, 931 (1996).

17 A. Elsheikh, D. Wang, M. Brown, P. Rama, M. Campanelli, and D. Pye, Current eye research 32, 11 (2007).

18 J. M. Dias and N. M. Ziebarth, Experimental eye research 115, 41 (2013).

19 A. Elsheikh and K. Anderson, Journal of the Royal Society Interface 2, 177 (2005).

20 S. Chang, J. Hjortdal, D. Maurice, and P. Pinsky, Investigative ophthalmology \& visual science 34, 1241 (1993).

21 M. W. Ko, L. K. Leung, D. C. Lam, and C. K. Leung, Acta ophthalmologica 91, e263 (2013).

22 M. W. Ko, L. K. Leung, and D. C. Lam, Medical engineering \& physics 36, 1115 (2014).

23 M. Tanter, D. Touboul, J.-L. Gennisson, J. Bercoff, and M. Fink, Medical Imaging, IEEE Transactions on 28, 1881 (2009).

24 K. W. Hollman, R. M. Shtein, S. Tripathy, and K. Kim, Ultrasound in medicine \& biology 39, 1451 (2013).

25 I. M. Beshtawi, R. Akhtar, M. C. Hillarby, C. O'Donnell, X. Zhao, A. Brahma, F. Carley, B. Derby, and $\mathrm{H}$. Radhakrishnan, Investigative ophthalmology \& visual science 54, 5273 (2013).

26 G. Scarcelli, R. Pineda, and S. H. Yun, Investigative ophthalmology \& visual science 53, 185 (2012).

27 D. A. Luce, Journal of Cataract \& Refractive Surgery 31, 156 (2005).

28 B. M. Fontes, R. Ambrósio Jr, G. C. Velarde, and W. Nosé, J Refract Surg 27, 209 (2011).

29 Y. Goldich, Y. Barkana, Y. Morad, M. Hartstein, I. Avni, and D. Zadok, Cornea 28, 498 (2009).

30 W. Lau and D. Pye, Investigative ophthalmology \& visual science 52, 3207 (2011).
31 M. Dubbelman, H. A. Weeber, R. G. Van Der Heijde, and H. J. Völker-Dieben, Acta Ophthalmologica Scandinavica 80, 379 (2002).

32 P. Rosales and S. Marcos, (2008).

33 A. Pérez-Escudero, C. Dorronsoro, L. Sawides, L. Remón, J. Merayo-Lloves, and S. Marcos, Investigative ophthalmology \& visual science 50, 4146 (2009).

34 I. Grulkowski, M. Gora, M. Szkulmowski, I. Gorczynska, D. Szlag, S. Marcos, A. Kowalczyk, and M. Wojtkowski, Optics express 17, 4842 (2009).

35 P. Targowski, M. Wojtkowski, A. Kowalczyk, T. Bajraszewski, M. Szkulmowski, and I. Gorczyńska, Optics Communications 229, 79 (2004).

36 D. Huang, Y. Li, and S. Radhakrishnan, Ophthalmology Clinics of North America 17, 1 (2004).

37 D. Alonso-Caneiro, K. Karnowski, B. J. Kaluzny, A. Kowalczyk, and M. Wojtkowski, Optics express 19, 14188 (2011).

38 C. Dorronsoro, D. Pascual, P. Pérez-Merino, S. Kling, and S. Marcos, Biomedical optics express 3, 473 (2012).

39 L.-K. Wang, Y.-P. Huang, L. Tian, C.-s. Kee, and Y.-P. Zheng, Ultrasonics (2016).

40 D. Luce, Investigative Ophthalmology \& Visual Science 46, 5009 (2005).

41 W. C. Young and R. G. Budynas, Roark's formulas for stress and strain (McGraw-Hill New York, 2002).

42 E. Reissner, J. Math. Phys 25, 279 (1946).

43 M. Zhang, Y. Zheng, and A. F. Mak, Medical Engineering and Physics 19, 512 (1997).

44 R. L. Norton, Machine design: an integrated approach (Pearson Prentice Hall New Jersey, 2006).

45 L. G. Portney and M. P. Watkins, Foundations of clinical research (Pearson/Prentice Hall, 1993).

46 N. E. K. Cartwright, J. R. Tyrer, and J. Marshall, Investigative ophthalmology \& visual science 52, 4324 (2011).

47 K. Wu, S. Li, A. Lo, A. Ngan, D. Wong, K. So, R. Ellis-Behnke, and B. Tang, Soft Materials 11, 244 (2013).

48 C. K.-S. Leung, C. Ye, and R. N. Weinreb, Investigative ophthalmology \& visual science 54, 2885 (2013). 